\title{
Epstein-Barr virus-associated lymphoproliferative disorder developed following autologous peripheral blood stem cell transplantation for relapsing Hodgkin's lymphoma
}

\author{
SAKURA IZUMIYA $^{1 *}$, MITSUAKI ISHIDA ${ }^{2 *}$, KEIKO HODOHARA ${ }^{1}$, \\ TAKASHI YOSHIDA ${ }^{2}$ and HIDETOSHI OKABE ${ }^{2}$ \\ Departments of ${ }^{1}$ Hematology, and ${ }^{2}$ Clinical Laboratory Medicine and Division of Diagnostic Pathology, \\ Shiga University of Medical Science, Otsu, Shiga, Japan \\ Received January 9, 2012; Accepted March 27, 2012
}

DOI: $10.3892 / \mathrm{ol} .2012 .670$

\begin{abstract}
Post-transplant lymphoproliferative disorders (PTLDs) are lymphoid or plasmacytic proliferations that develop as a consequence of immunosuppression in a recipient of a solid organ, bone marrow or stem cell allograft. The development of PTLDs is usually associated with Epstein-Barr virus (EBV) and the disorder is also termed EBV-associated lymphoproliferative disorder(LPD). The development of PTLD is a rare complication in autologous bone marrow/peripheral blood stem cell transplantation. In the present study, we report a case of EBV-associated LPD which developed following autologous peripheral blood stem cell transplantation for relapsing Hodgkin's lymphoma. A 51-year-old male presented with swelling of the left cervical lymph nodes. A biopsy revealed nodular sclerosis classical Hodgkin's lymphoma. Following four courses of ABVd (adriamycin, bleomycin, vinblastine, dacarbazine) therapy, the Hodgkin's lymphoma relapsed. CHASE (cyclophosphamide, etoposide, cytarabine, dexamethasone) therapy and autologous peripheral blood stem cell transplantation were performed. In the 128 days following the transplantation, lymph node swelling was noted and a biopsy specimen demonstrated EBV-associated LPD. The serum copy number of EBV-DNA was $2.7 \times 10^{3}$ copies $/ \mathrm{ml}$. The occurrence of EBV-associated LPD may be on the rise due to the increased number of patients undergoing immunosuppression therapy. The measurement of the serum EBV-DNA copy
\end{abstract}

Correspondence to: Dr Mitsuaki Ishida, Department of Clinical Laboratory Medicine and Division of Diagnostic Pathology, Shiga University of Medical Science, Tsukinowa-cho, Seta, Otsu, Shiga 520-2192, Japan

E-mail: mitsuaki@belle.shiga-med.ac.jp

${ }^{*}$ Contributed equally

Key words: Epstein-Barr virus, lymphoproliferative disorder, Hodgkin's lymphoma, autologous peripheral blood stem cell transplantation number and the detection of EBV-infected atypical lymphocytes using in situ hybridization are significant in establishing an early accurate diagnosis and initiating the correct treatment for EBV-associated LPD in patients with immunosuppression.

\section{Introduction}

Post-transplant lymphoproliferative disorders (PTLDs) are defined as lymphoid or plasmacytic proliferations that develop as a consequence of immunosuppression in a recipient of a solid organ, bone marrow or stem cell allograft (1) and are classified as immunodeficiency-associated lymphoproliferative disorders (LPDs) in the WHO classification (1). The development of PTLD is usually associated with Epstein-Barr virus (EBV) and the disorder is also termed EBV-associated LPD. The risk of developing PTLD varies; patients receiving renal allografts have the lowest frequency of PTLD $(<1 \%)$, those with hepatic and cardiac allografts have an intermediate risk (1-2\%) and those with heart-lung, lung or intestinal allografts have the highest frequency (>5\%) (2-4). Peripheral blood, stem cell and bone marrow allograft recipients have a low risk of PTLD $(\sim 1 \%)$ and PTLD is rare following autologous bone marrow transplantation (5-11).

In the present study, we report a case of EBV-associated LPD that developed following autologous peripheral blood stem cell transplantation for relapsing Hodgkin's lymphoma.

\section{Materials and methods}

Case report. A 51-year-old Japanese male without a noteworthy past or family history presented with a painless tumor in the left neck at a dermatological clinic. The dermatologist observed swelling of the lymph nodes in his left neck and the patient was referred to the Department of Hematology of our hospital (Shiga University of Medical Sciences, Shiga, Japan).

A physical examination revealed several swollen lymph nodes in the left neck of the patient, however, no palpable superficial lymph nodes, with the exception of those in the left neck, or hepatosplenomegaly were detected. Laboratory tests revealed slightly elevated lactate dehydrogenase (333 U/1; range, 119-229) and elevated soluble IL-2 receptor (sIL-2R) 

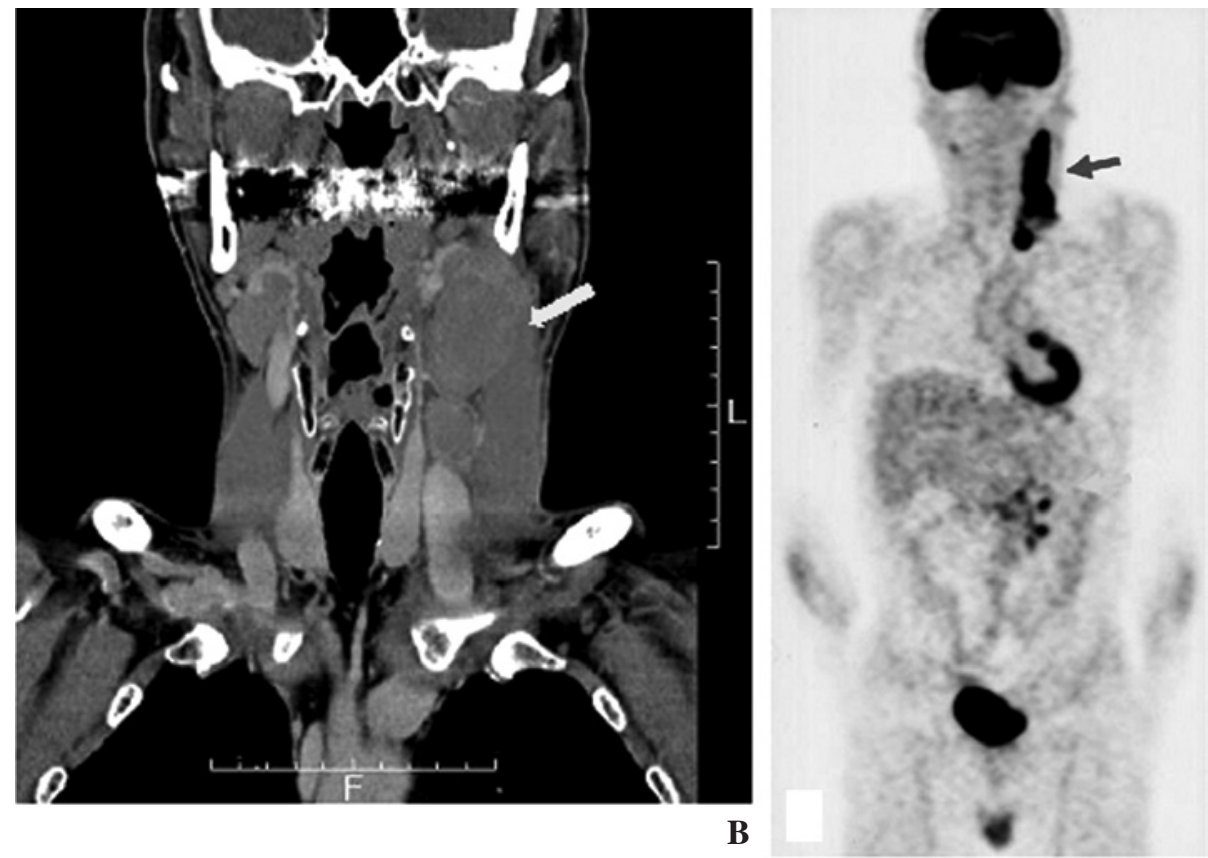

Figure 1. Computed tomography and positron emission tomography. (A) Computed tomography scan showing swelling of the left cervical lymph nodes (arrow). (B) Positron emission tomography scan showing accumulation in the left cervical and mesenteric lymph nodes (arrows).

(2,070 U/ml; range, 124-466). In addition, the EBV infection status of the patient was prior infection. Computed tomography scan (CT) revealed several swollen lymph nodes with a maximum diameter of $5 \times 3 \mathrm{~cm}$ in the left neck of the patient and positron emission tomography (PET) scan revealed an accumulation in the lymph nodes of the left neck and mesentery (Fig. 1).

Treatment. Clinically, malignant lymphoma was suspected and the biopsy specimen of the left cervical lymph node revealed nodular sclerosis classical Hodgkin's lymphoma (stage IIIa). Following therapy with ABVd (adriamycin, bleomycin, vinblastine, dacarbazine), the size of the cervical lymph nodes was reduced and the concentration of sIL-2R also decreased (710 U/ml). Nevertheless, following 4 courses of $\mathrm{ABVd}$ therapy, PET scan revealed swelling of the left cervical lymph nodes of the patient and a repeated biopsy revealed relapsing Hodgkin's lymphoma.

The patient underwent CHASE (cyclophosphamide, etoposide, cytarabine, dexamethasone) therapy and autologous peripheral blood stem cell transplantation was performed. Fever and skin eruption appeared following transplantation and steroid therapy was added. The fever went into remission, but the skin eruption was prolonged. In the 128 days following transplantation, CT scan revealed swelling of the lymph nodes of the bilateral axilla as well as the inguinal and para-aortal nodes and a biopsy of the inguinal lymph node was performed. The serum copy number of EBV-DNA was $2.7 \times 10^{3}$ copies/ml at that time. R-CHOP (rituximab, cyclophosphamide, hydroxydaunorubicin, oncovin, prednisolone) therapy was performed. Following two courses of R-CHOP therapy, the swelling of the lymph nodes was reduced and the serum copy number of EBV-DNA was below the detection sensitivity.
In the 270 days following transplantation, the fever reappeared and chest CT scan revealed ground glass opacity in the bilateral lungs. A diagnosis of cytomegalovirus pneumonia was made, as the serum C7-HRP was positive. Anti-cytomegalovirus therapy was performed; however, the patient succumbed to respiratory dysfunction on the 369th day following transplantation.

Tissue samples and staining. Formalin-fixed, paraffinembedded tissue blocks of the cervical and inguinal lymph nodes were cut into $3 \mu \mathrm{m}$ sections, deparaffinized and rehydrated. Each section was stained with hematoxylin and eosin and then used for immunostaining and in situ hybridization. Immunohistochemical and in situ hybridization analyses were performed using an autostainer (XT system Benchmark, Ventana Medical System, Tucson, AZ, USA) according to the manufacturer's instructions. The following primary antibodies were used: a mouse monoclonal antibody against CD3 (PS1, Novocastra Laboratories, Ltd., Newcastle upon Tyne, UK), a mouse monoclonal antibody against CD8 (1A5, Novocastra), a mouse monoclonal antibody against CD15 (C3D1, DAKO Cytomation, Glostrup, Denmark), a mouse monoclonal antibody against CD20 (L26, Novocastra) and a mouse monoclonal antibody against CD30 (1G12, Novocastra). For in situ hybridization, an INFORM EBER (EBV-encoded early RNA) probe (Ventana Medical System) was used.

\section{Results}

Biopsy specimen of the cervical lymph nodes. The histopathological analysis of the first biopsy specimen revealed that normal lymph node architecture was not preserved and that sclerotic fibrous tissue divided the lymph nodes into nodules (Fig. 2). In the nodules, there were atypical large lymphocytes 


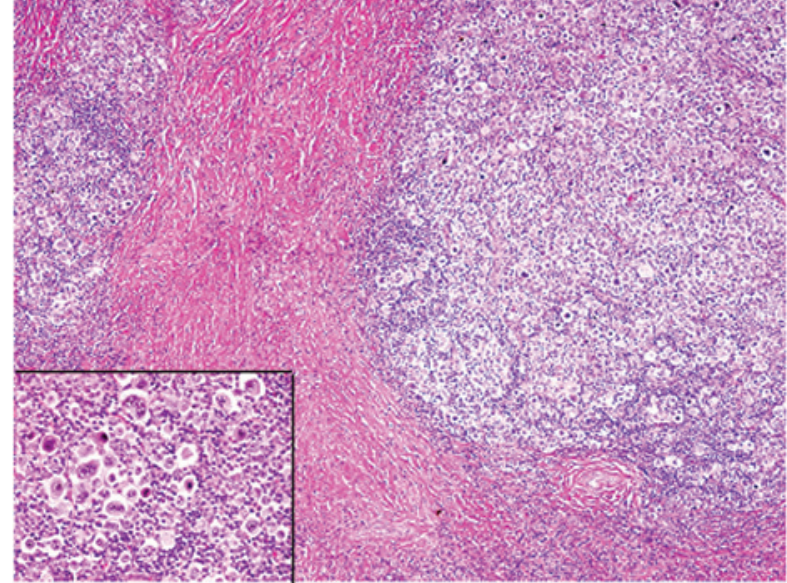

Figure 2. Histopathological findings of the first biopsy of the cervical lymph node. Sclerotic fibrous tissue divides the lymph node into nodules and a number of atypical large lymphocytes are present in the nodules. Numerous lacuna and Hodgkin's cells are present and occasionally Reed-Sternberg cells are also observed (inset). Hematoxylin and eosin stain; original magnification, $\mathrm{x} 40$ (inset, $\mathrm{x} 200$ ).

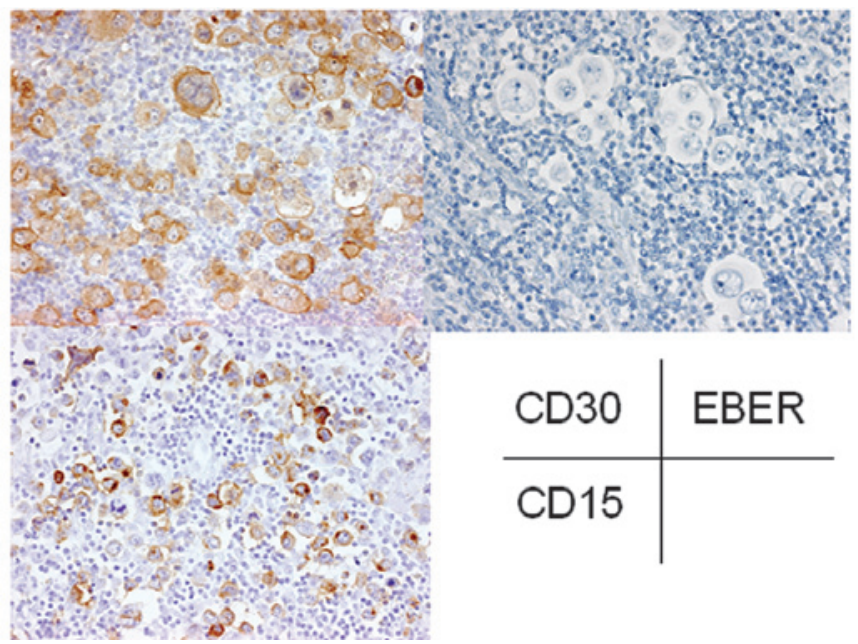

Figure 3. Immunohistochemical and in situ hybridization findings of the first biopsy of the cervical lymph node. Large atypical lymphocytes are positive for CD15 and CD30, but no EBER-positive atypical lymphocytes are present (original magnification, x400). EBER, EBV-encoded early RNA.

(namely Hodgkin's cells) with single large nuclei, prominent nucleoli and large amounts of slightly eosinophilic cytoplasm; lacuna cells, which had clear cytoplasm with spider-web-like extensions to the cell membrane, folded nuclear membranes and less conspicuous nucleoli, were scattered and mixed with numerous small-sized lymphocytes, histiocytes and eosinophils (Fig. 2, inset). Reed-Sternberg cells, which had two large nuclei and a prominent nucleolus, were also observed (Fig. 2, inset). Immunohistochemical analyses demonstrated that these atypical large lymphocytes were positive for CD15 and CD30 (Fig. 3), but negative for CD3 and CD20. EBER was not detected in these atypical lymphocytes by in situ hybridization (Fig. 3). These findings led to the ultimate diagnosis of nodular sclerosis classical Hodgkin's lymphoma.

The histopathological and immunohistochemical findings of the repeated biopsy specimen of the cervical lymph

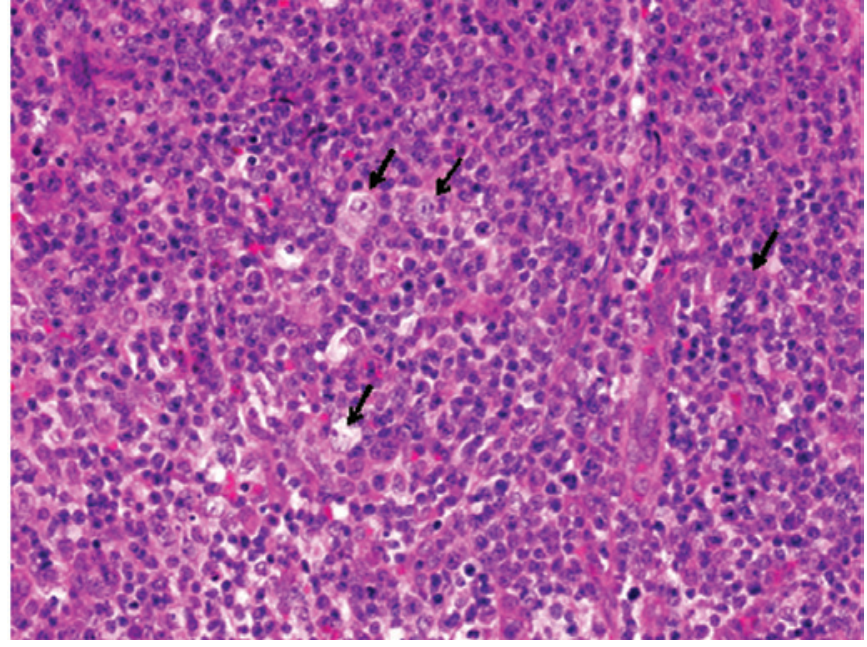

Figure 4. Histopathological findings of the inguinal lymph node biopsy. Atypical large lymphocytes with prominent nucleoli are scattered among numerous small to medium-sized lymphocytes (arrows). Hematoxylin and eosin stain; original magnification, $\mathrm{x} 100$.

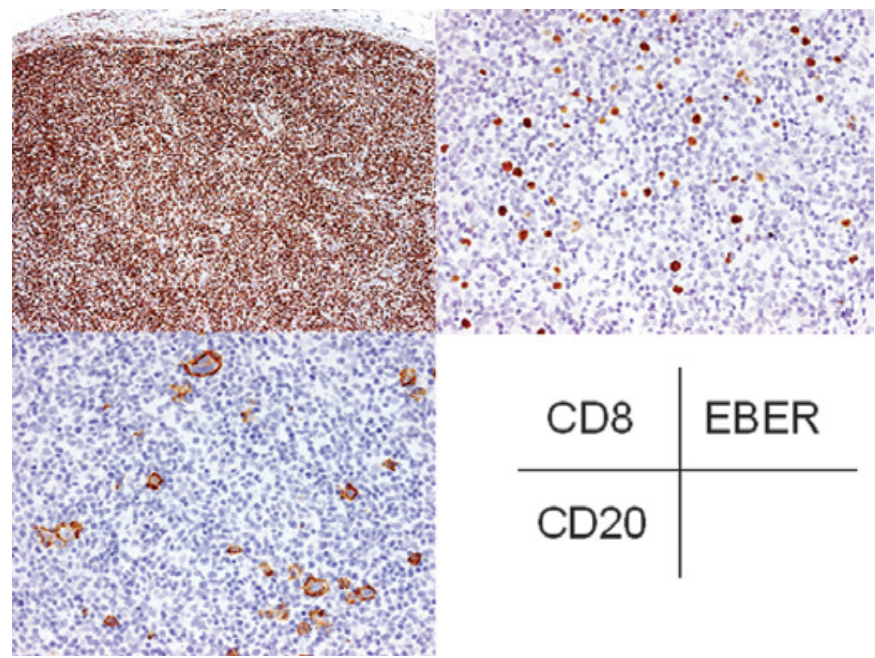

Figure 5. Immunohistochemical and in situ hybridization findings of the inguinal lymph node biopsy. CD20 and EBER are expressed in the atypical large lymphocytes. Small lymphocytes are positive for CD8. Original magnification, x40 (CD8); x100 (CD20, EBER). EBER, EBV-encoded early RNA.

node were the same as those of the first biopsy specimen as described above.

Biopsy specimen of the inguinal lymph node. The histopathological analyses revealed that normal lymph node architecture was not preserved and that atypical large lymphocytes with prominent nucleoli were scattered among numerous small to medium-sized lymphocytes (Fig. 4). Neither typical lacuna cells nor Reed-Sternberg cells were observed.

Immunohistochemically, CD20 was expressed in the atypical large lymphocytes and CD30 was also expressed in most of these cells (Fig. 5). However, CD15 was not expressed in these cells. The small to medium-sized lymphocytes were positive for CD3 and CD8 (Fig. 5). EBER was detected in the atypical large lymphocytes by in situ hybridization. Therefore, a diagnosis of EBV-associated LPD was made. 


\section{Discussion}

PTLD is classified into four categories according to the WHO classification (1): early lesions, polymorphic PTLD, monomorphic PTLD and classical Hodgkin's lymphoma-type PTLD. The histopathological and immunohistochemical features of EBV-associated LPD of the present case corresponded to polymorphic PTLD (1), as EBER- and CD20-positive atypical large lymphocytes were scattered in numerous CD8-positive $\mathrm{T}$ lymphocytes accompanying lymph node architectural effacement. A differential diagnosis of relapsing Hodgkin's lymphoma was considered. However, CD15 was not expressed in atypical lymphocytes and typical lacuna cells and ReedSternberg cells were not observed in the present case. Therefore, the possibility of relapsing Hodgkin's lymphoma was excluded.

Most individuals become infected with EBV and the virus persists within the body throughout life in resting memory B cells. T lymphocytes control proliferating EBV-infected $B$ cells. In a setting of decreased or impaired T-cell immune surveillance for EBV-infected B cells, as observed during immunosuppression in connection with the transplantation of solid organs or bone marrow, the unchecked replication of B lymphocytes may lead to polyclonal B-cell hyperplasia and/or the monoclonal proliferation of B cells (12). In the present case, the decreased or impaired immune surveillance for EBV-infected B cells due to chemotherapy and/or blood stem cell transplantation for relapsing Hodgkin's lymphoma may be correlated with the development of EBV-associated LPD.

The development of PTLD is a rare complication in autologous bone marrow/peripheral blood stem cell transplantation (5-11). Nash et al reported that 2 of 56 patients (3.6\%) with severe autoimmune diseases developed EBV-associated LPD following autologous stem cell transplantation and that the $\mathrm{CD}^{+}$cell count was $0 / \mu 1$ in these two cases (5). In addition, Powell et al reported that 5 of 156 patients (3.5\%) with neuroblastoma developed EBV-associated LPD following autologous peripheral blood stem cell transplantation (6). However, only two case reports of EBV-associated LPD which developed following autologous bone marrow transplantation for Hodgkin's lymphoma have been published previously $(8,9)$; one case was polymorphous B-cell PTLD (8), as was the present case, and the other was T-cell PTLD (9).

Early detection of the development of EBV-associated LPD is a significant clinical problem. The monitoring of EBV-DNA load in the peripheral blood is a useful method to detect the development of EBV-associated LPD, especially in high-risk patients $(13,14)$. Moreover, EBV-associated LPD may occur in the gastrointestinal tract, lung and liver, as well as in lymph nodes (1). Therefore, detection of EBER-positive atypical lymphocytes in the biopsy specimen may lead to the diagnosis of EBV-associated LPD.

In conclusion, we report a case of EBV-associated LPD that developed following autologous peripheral blood stem cell transplantation for relapsing Hodgkin's lymphoma. The occurrence of EBV-associated LPD may be on the rise due to the increased number of patients undergoing immunosuppression therapy (15). Therefore, the measurement of serum EBV-DNA copy number and detection of EBV-infected atypical lymphocytes by in situ hybridization are significant in making an early accurate diagnosis and initiating the correct treatment of EBV-associated LPD in patients undergoing immunosuppression.

\section{References}

1. Swerdlow SH, Webber SA, Chadburn A and Ferry JA: Posttransplant lymphoproliferative disorders. In: WHO Classification of Tumours of Haematopoietic and Lymphoid Tissues. Swerdlow SH, Campo E, Harris NL, et al (eds). IARC, Lyon, pp343-349, 2008.

2. Opelz G and Döhler B: Lymphomas after solid organ transplantation: a collaborative transplant study report. Am J Transplant 4: 222-230, 2003.

3. Bakker NA, van Imhoff GW, Verschuuren EA, et al: Early onset post-transplant lymphoproliferative disease is associated with allograft localization. Clin Transplant 19: 327-334, 2005.

4. Caillard S, Lelong C, Pessione F and Moulin B: Post-transplant lymphoproliferative disorders occurring after renal transplantation in adults: report of 230 cases from the French Registry. Am J Transplant 6: 2735-2742, 2006.

5. Nash RA, Dansey R, Storek J, et al: Epstein-Barr virusassociated posttransplantation lymphoproliferative disorder after high-dose immunosuppressive therapy and autologous CD34-seletcted hematopoietic stem cell transplantation for severe autoimmune diseases. Biol Blood Marrow Transplant 9: 583-591, 2003.

6. Powell JL, Bunin NJ, Callahan C, Aplenc R, Griffin G and Grupp SA: An unexpectedly high incidence of Epstein-Barr virus lymphoproliferative disease after $\mathrm{CD} 4^{+}$selected autologous peripheral blood stem cell transplant in neuroblastoma. Bone Marrow Transplant 33: 651-657, 2004

7. Peniket AJ, Perry AR, Williams CD, et al: A case of EBV-associated lymphoproliferative disease following high-dose therapy and CD34-purified autologous peripheral blood progenitor cell transplantation. Bone Marrow Transplant 22: 307-309, 1998.

8. Hauke RJ, Greiner TC, Smir BN, et al: Epstein-Barr virusassociated lymphoproliferative disorder after autologous bone marrow transplantation: report of two cases. Bone Marrow Transplant 21: 1271-1274, 1998.

9. Yufu Y, Kimura M, Kawano R, et al: Epstein-Barr virus-associated $\mathrm{T}$ cell lymphoproliferative disorder following autologous blood stem cell transplantation for relapsed Hodgkin's disease. Bone Marrow Transplant 26: 1339-1341, 2000.

10. Lones MA, Kirov I, Said JW, Shintaku IP and Neudorf S: Post-transplant lymphoproliferative disorder after autologous peripheral stem cell transplantation in a pediatric patient. Bone Marrow Transplant 26: 1021-1024, 2000.

11. Heath JA, Broxson EH Jr, Dole MG, et al: Epstein-Barr virusassociated lymphoma in a child undergoing an autologous stem cell rescue. J Pediatr Hematol Oncol 24: 160-163, 2002.

12. List AF, Greco FA and Vogler LB: Lymphoproliferative diseases in immunocompromised hosts: the role of Epstein-Barr virus. J Clin Oncol 5: 1673-1689, 1987.

13. Shaffer DR, Rooney CM and Gottschalk S: Immunotherapeutic options for Epstein-Barr virus-associated lymphoproliferative disease following transplantation. Immunotherapy 2: 663-671, 2010.

14. Meijer E and Cornelissen JJ: Epstein-Barr virus-associated lymphoproliferative disease after allogenic haematopoietic stem cell transplantation: molecular monitoring and early treatment of high-risk patients. Curr Opin Hematol 15: 576-585, 2008.

15. Ohta M, Taga T, Nomura A, et al: Epstein-Barr virus-related lymphoproliferative disorder, cytomegalovirus reactivation, and varicella zoster virus encephalitis during treatment of medulloblastoma. J Med Virol 83: 1582-1584, 2011. 